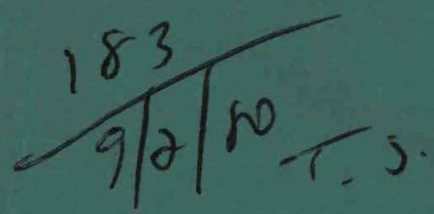

\title{
ON CARRIER FACILITATED TRANSPORT THROUGH MEMBRANES
}

by

\author{
Hans G. Kaper, Gary K. Leaf, \\ and Bernard J. Matkowsky
}

ARGONNE NATIONAL LABORATORY, ARGONNE, ILLINOIS

Prepared for the U. S. DEPARTMENT OF ENERGY under Contract W-31-109-Eng-38 


\section{DISCLAIMER}

This report was prepared as an account of work sponsored by an agency of the United States Government. Neither the United States Government nor any agency Thereof, nor any of their employees, makes any warranty, express or implied, or assumes any legal liability or responsibility for the accuracy, completeness, or usefulness of any information, apparatus, product, or process disclosed, or represents that its use would not infringe privately owned rights. Reference herein to any specific commercial product, process, or service by trade name, trademark, manufacturer, or otherwise does not necessarily constitute or imply its endorsement, recommendation, or favoring by the United States Government or any agency thereof. The views and opinions of authors expressed herein do not necessarily state or reflect those of the United States Government or any agency thereof. 


\section{DISCLAIMER}

Portions of this document may be illegible in electronic image products. Images are produced from the best available original document. 
The facilities of Argonne National Laboratory are owned by the United States Government. Under the terms of a contract (W-31-109-Eng-38) among the U. S. Department of Energy, Argonne Universities Association and The University of Chicago, the University employs the staff and operates the Laboratory in accordance with policies and programs formulated, approved and reviewed by the Association.

\section{MEMBERS OF ARGONNE UNIVERSITIES ASSOCIATION}

The University of Arizona

Carnegie-Mellon University

Case Western Reserve University

The University of Chicago

University of Cincinnati

Illinois Institute of Technology

University of Illinois

Indiana University

The University of Iowa

Iowa State University
The University of Kansas

Kansas State University

Loyola University of Chicago

Marquette University

The University of Michigan

Michigan State University

University of Minnesota

University of Missouri

Northwestern University

University of Notre Dame
The Ohio State University

Ohio University

The Pennsylvania State University

Purdue University

Saint Louis University

Southern Illinois University

The University of Texas at Austin

Washington University

Wayne State University

The University of Wisconsin-Madison

-NOTICE

This report was prepared as an account of work sponsored by an agency of the United States Government. Neither the United States Government or any agency thereof, nor any of their employees, make any warranty, express or implied, or assume any legal liability or responsibility for the accuracy, completeness, or usefulness of any information, apparatus, product, or process disclosed, or represent that its use would not infringe privately owned rights. Reference herein to any specific commercial product, process, or service by trade name, mark, manufacturer, or otherwise, does not necessarily constitute or imply its endorsement, recommendation, or favoring by the United States Government or any agency thereof. The views and opinions of authors expressed herein do not necessarily state or reflect those of the United States Government or any agency thereof.

Printed in the United States of America

Available from

National Technical Information Service

U. S. Department of Commerce

5285 Port Royal Road

Springfield, VA 22161

NTIS price codes

Printed copy: A03

Microfiche copy: A01 


\section{ARGONNE NATIONAL LABORATORY \\ 9700 South Cass Avenue Argonne, Illinois 60439}

by

ON CARRIER FACILITATED TRANSPORT THROUGH MEMBRANES

Hans G. Kaper, Gary K. Leaf, Bernard J. Matkowsky ${ }^{*}$

Applied Mathematics Division

June 1980

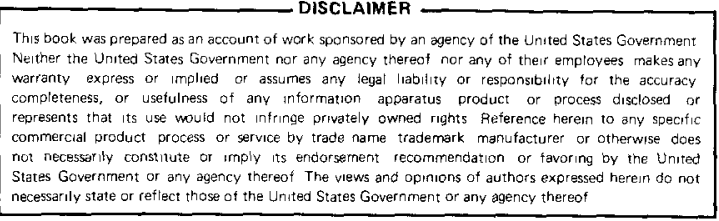

* Permanent address: Department of Engineering Sciences and Applied Mathematics, Northwestern University, Evanston, IL 60201. 


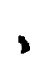


TABLE OF CONTENTS

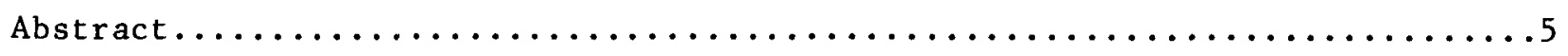

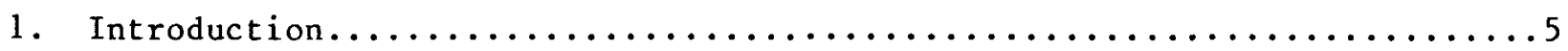

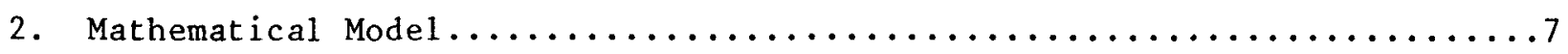

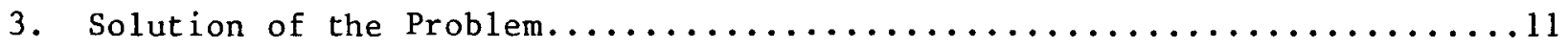

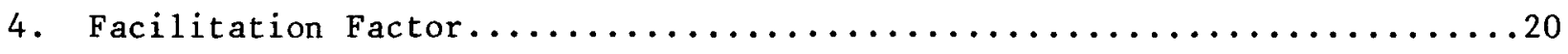

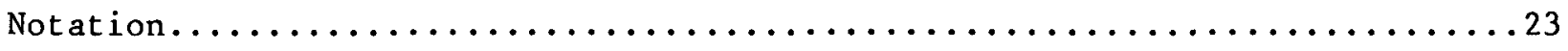

References............................................ 24 

ON CARRIER FACILITATED TRANSPORT

THROUGH MEMBRANES

by

Hans G. Kaper, Gary K. Leaf, Bernard J. Matkowsky

\section{Abstract}

Facilitated transport is a process whereby the diffusion of a solute across a membrane is chemically enhanced. In this report an analysis is given of a facilitated transport system involving a volatile species $A$ which reacts with a nonvolatile carrier species $B$ to form the nonvolatile product $A B$. The species $A$ is transported across the membrane by ordinary diffusion, as well as by the diffusion of the product $A B$. It is assumed that the reaction rates are large, so the reactions are confined mostly to thin boundary layers near the surfaces of the membrane. The method of matched asymptotic expansions is used to derive the asymptotic solution of the nonlinear boundary value problem governing equilibrium. The effect of various parameters on the facilitation factor is analyzed in detail.

\section{Introduction}

Facilitated transport is a process whereby the diffusion of a solute across a membrane is chemically enhanced. The phenomenon of facilitated transport is observed, for example, when a volatile species A diffuses across a membrane and the membrane contains a nonvolatile carrier species $B$ which reacts reversibly with $A$ to form the nonvolatile complex $A B$. In addition to the ordinary diffusion of $A$, we have the diffusion of the complex $A B$ across the membrane. The carrier B cannot leak out of the membrane, so the total amount of $B$ is conserved and, to balance the diffusion of $A B$, the carrier $B$ diffuses in a direction opposite to that of $A$ and $A B$. This circulation of $B$ enhances the transport of $A$ across the membrane. Near the surface of higher concentration of $A$ the reaction of $A$ and $B$ is such that the complex $A B$ is formed, while near the opposite surface the complex $A B$ dissociates into its components $A$ and $B$. After the dissociation, $A$ leaves the membrane and $B$ diffuses back through the membrane to form additional products $A B$. The flux of $A$ is thus facilitated by the presence of the carrier species $B$. This mechanism of facilitated transport is, in fact, used in separation and purification processes -- for example, to separate hexene from heptane (cf. 
Matulevicius and $\mathrm{Li}$ [1]). It is also assumed to play an important role in transport phenomena in biological membranes, cf. Murray [2].

The phenomenon of facilitated transport was first observed in 1959 by Scholander [3] and Wittenberg [4]. It was studied by Ward [5] who formulated a mathematical model and compared the results of numerical calculations with experimental data for the facilitated transport of nitric oxide in a ferrous chloride solution. Ward also gave an expression for the facilitation factor in the case of equal diffusion coefficients and infinite reaction rates. The expression was derived by selectively applying boundary conditions to the resulting algebraic equations, in an ad hoc manner. Smith, Meldon and Colton [6] considered the same mathematical model and found asymptotic solutions corresponding to membranes that are either very thin or very thick. They recognized that the latter case leads to a singularly perturbed boundary value problem. Assuming equal diffusion coefficients for the nonvolatile species they used the method of matched asymptotic expansions to construct an approximate solution valid for large membrane thicknesses and carrier-dominated diffusion. Goddard, Schultz and Bassett [7] also employed the method of matched asymptotic expansions to consider the facilitated transport problem with large reaction rates and equal diffusion coefficients. This work is reviewed in the article by Goddard, Schultz and Suchdeo [8], and in the monograph by Aris [9], where an excellent description of facilitated transport can be found.

In this report we discuss the carrier facilitated transport problem described earlier. As in [6]-[9], we assume a fast reaction mechanism, so the reactions are confined mostly to thin boundary layers near the surfaces of the membrane and chemical equilibrium pertains throughout most of the interior of the membrane. We make no assumptions about the diffusivities or any of the other parameters that enter the model, except that they are constant -- but not necessarily equal -- throughout the membrane. Our results are asymptotically complete to lowest order for large reaction rates. In the case of equal diffusion coefficients for the nonvolatile species our solution reduces to the solution found by Goddard, Schultz and Bassett [7] which was also given by Ward [5]. In the final section we give a general appraisal of the effect of the various parameters of the problem upon the facilitation factor. 


\section{Mathematical Mode1}

We consider the steady molecular diffusion of a chemical species A through a membrane. The membrane has the form of a plane slab of finite thickness L. Inside the membrane the species A undergoes a reversible chemical reaction with a second species $B$ to form a complex species $A B$, say,

$$
\mathrm{A}+\mathrm{B} \doteqdot \mathrm{AB}
$$

The carrier $B$ and the complex $A B$ diffuse freely inside the membrane, but cannot leak out of it. Thus, only the species $A$ is transferred through the membrane, either by ordinary diffusion or by carrier-mediated transport.

We assume that the temperature is uniform throughout the membrane, and that the diffusion processes are governed by Fick's law with constant (but not necessarily equal) diffusion coefficients. If we choose the $x^{*}$-axis perpendicular to the membrane, with the membrane surfaces at $x^{*}=0$ and $x^{*}=L$, then the molar concentrations $C$ satisfy the differential equations

$$
\begin{gathered}
D_{A} \frac{d^{2} C_{A}}{d x^{2}}=k_{1} C_{A} C_{B}-k_{2} C_{A B}, \\
D_{B} \frac{d^{2} C_{B}}{d x^{*}}=k_{1} C_{A} C_{B}-k_{2} C_{A B}, \\
D_{A B} \frac{d^{2} C_{A B}}{d x *^{2}}=-k_{1} C_{A} C_{B}+k_{2} C_{A B},
\end{gathered}
$$

for $0<x^{*}<L$, and the boundary conditions

$$
\begin{aligned}
& C_{A}(0)=C_{A}^{0}, \quad C_{A}(L)=C_{A}^{L}, \\
& \frac{\mathrm{dC}_{B}}{d x^{*}}(0)=\frac{\mathrm{dC}_{B}}{d x^{*}}(L)=0, \\
& \frac{\mathrm{dC}_{\mathrm{AB}}}{\mathrm{dx^{* }}(0)}=\frac{\mathrm{dC}_{\mathrm{AB}}}{\mathrm{dx^{* }}}(\mathrm{L})=0 .
\end{aligned}
$$

Here, the D's denote the diffusion coefficients of the various species; $k_{1}$ and $k_{2}$ are the reaction rates associated with the forward $(\rightarrow)$ and backward $(*)$ reaction (1), respectively; $C_{A}^{0}$ and $C_{A}^{L}$ are the specified molar concentrations of species $A$ at the faces $x^{*}=0$ and $x^{*}=L$ of the membrane. The boundary 
value problem (2),(3) is to be solved subject to the stoichiometric constraint

$$
\frac{1}{\mathrm{~L}} \int_{0}^{\mathrm{L}}\left(\mathrm{C}_{\mathrm{B}}+\mathrm{C}_{\mathrm{AB}}\right) \mathrm{d} \mathrm{x}^{*}=\mathrm{C} \text {, }
$$

where $\mathrm{C}$ is a specified molar concentration. This constraint fixes the total amount of the nontransferable species $B$ and $A B$ present in the membrane.

The quantity of interest is the net molar flux of species A across the membrane, which is given by

$$
\mathrm{N}_{\mathrm{A}}\left(\mathrm{x}^{*}\right)=-\left(\mathrm{D}_{\mathrm{A}} \frac{\mathrm{dC}}{\mathrm{dx^{* }}}+\mathrm{D}_{\mathrm{AB}} \frac{\mathrm{dC}_{\mathrm{AB}}}{\mathrm{dx^{* }}}\right), \quad 0 \leq \mathrm{x}^{*} \leq \mathrm{L} .
$$

It follows from (2a) and (2c) that the quantity $D_{A} C_{A}+D_{A B} C_{A B}$ varies linearly across the membrane. Hence, $\mathrm{N}_{A}$ is constant; its value can be expressed in terms of the concentration differences of the species $A$ and $A B$ at the surfaces of the membrane,

$$
\mathrm{N}_{\mathrm{A}}=\frac{\mathrm{D}_{\mathrm{A}}}{\mathrm{L}}\left(\mathrm{C}_{\mathrm{A}}^{0}-\mathrm{C}_{\mathrm{A}}^{\mathrm{L}}\right)+\frac{\mathrm{D}_{\mathrm{AB}}}{\mathrm{L}}\left(\mathrm{C}_{\mathrm{AB}}(0)-\mathrm{C}_{\mathrm{AB}}(\mathrm{L})\right) .
$$

The first term in this expression represents the contribution due to ordinary diffusion of the species A, whereas the second term represents the contribution due to carrier-mediated transport of $\mathrm{A}$ in the form of the complex $\mathrm{AB}$. It follows from (5) that we are interested in finding the quantity $C_{A B}(0)-C_{A B}(L)$.

We introduce dimensionless variables,

$$
\begin{array}{rlrl}
\mathrm{x} & =\mathrm{x}^{*} / \mathrm{L}, \\
\mathrm{u}(\mathrm{x}) & =\mathrm{C}_{\mathrm{A}}\left(\mathrm{x}^{*}\right) / \mathrm{C}, \\
\mathrm{v}(\mathrm{x}) & =\mathrm{C}_{\mathrm{AB}}\left(\mathrm{x}^{*}\right) / \mathrm{C}, \\
\mathrm{w}(\mathrm{x}) & =\mathrm{C}_{\mathrm{B}}\left(\mathrm{x}^{*}\right) / \mathrm{C}, \\
\gamma_{0} & =\mathrm{C}_{\mathrm{A}}^{0} / \mathrm{C}, & \gamma_{1}=\mathrm{C}_{\mathrm{A}}^{\mathrm{L}} / \mathrm{C}, \\
\kappa_{1} & =\mathrm{k}_{1} \mathrm{CL}^{2} / \mathrm{D}_{\mathrm{A}}, \quad \mathrm{K}_{2}=\mathrm{k}_{2} \mathrm{~L}^{2} / \mathrm{D}_{\mathrm{A}}, \\
\delta & =\mathrm{D}_{\mathrm{AB}} / \mathrm{D}_{\mathrm{A}}, & \mathrm{d}=\mathrm{D}_{\mathrm{AB}} / \mathrm{D}_{\mathrm{B}} .
\end{array}
$$

Then we have the following boundary value problem for the functions $u$, $v$, and w, 


$$
\begin{aligned}
u^{\prime \prime} & =\kappa_{1} u w-\kappa_{2} v, \\
\delta v^{\prime \prime} & =-\kappa_{1} u w+\kappa_{2} v, \\
(\delta / d) w^{\prime \prime} & =\kappa_{1} u w-\kappa_{2} v,
\end{aligned}
$$

for $0<x<1$, and

$$
\begin{aligned}
\mathrm{u}(0) & =\gamma_{0}, \quad u(1)=\gamma_{1}, \\
\mathrm{v}^{\prime}(0) & =\mathrm{v}^{\prime}(1)=0, \\
\mathrm{w}^{\prime}(0) & =\mathrm{w}^{\prime}(1)=0,
\end{aligned}
$$

subject to the constraint

$$
\int_{0}^{1}(v+w) d x=1
$$

In terms of the dimensionless variables the quantity of interest, $\mathrm{N}_{\mathrm{A}}$, is given by

$$
N_{A}=\frac{D_{A} C}{L}\left\{\gamma_{0}-\gamma_{1}+\delta[v(0)-v(1)]\right\} \text {. }
$$

We recall that $u, v$, and $w$, representing dimensionless concentrations, are nonnegative functions on their entire domain of definition, $0 \leq \mathrm{x} \leq 1$.

By eliminating the variable w we reformulate the problem $(6),(7),(8)$ as a constrained boundary value problem for the functions $u$ and v. From (6b) and (6c) it follows that $w+d v$ is a linear function of $x$. According to (7b), the derivative of this function vanishes at the endpoints. Hence, $w+d v$ is a constant, $\alpha$ say, so

$$
w(x)=\alpha-d v(x), \quad 0 \leq x \leq 1
$$

Substituting this expression in (8) we see that the constant $\alpha$ can be expressed in terms of $v$,

$$
\alpha=1+(d-1) \bar{v},
$$

where

$$
\bar{v}=\int_{0}^{1} v(x) d x
$$


As $w(x)+d v(x) \geq 0$, it follows from (10) that the constant $\alpha$ is nonnegative. From (11) it follows that $\alpha \geq 1$ whenever $d \geq 1$. of course, $\bar{v}$ depends implicitly on the parameter $\alpha$, so eq. (11a) represents a (nonlinear) constraint which determines $\alpha$.

Using (10) to eliminate $w$, we obtain a boundary value problem for the pair of functions $(u, v)$,

$$
\begin{aligned}
u^{\prime \prime} & =\kappa_{1} \alpha_{u}-\kappa_{2} v-\kappa_{1} d u v, \\
\delta v^{\prime \prime} & =-\kappa_{1} \alpha_{u}+\kappa_{2} v+\kappa_{1} d u v,
\end{aligned}
$$

for $0<x<1$, subject again to the boundary conditions ( $7 a)$ and (7b), and the constraint (11). We will solve this problem assuming that the dimensionless reaction rates $\kappa_{1}$ and $\kappa_{2}$ are both large and of the same order of magnitude. That is, we assume that there exists a small positive parameter $\varepsilon, 0<\varepsilon \ll 1$, such that

$$
\kappa_{1}=\frac{\sigma_{1}}{\varepsilon^{2}}, \quad \kappa_{2}=\frac{\sigma_{2}}{\varepsilon^{2}},
$$

where the ratio

$$
\sigma=\sigma_{2} / \sigma_{1}
$$

is a $0(1)$-quantity compared to $\varepsilon$. Thus, we consider the equations

$$
\begin{aligned}
\varepsilon^{2} u^{\prime \prime} & =\sigma_{1} \alpha_{u}-\sigma_{2} v-\sigma_{1} \text { duv }, \\
\varepsilon^{2} \delta_{v} \prime & =-\sigma_{1} \alpha_{u}+\sigma_{2} v+\sigma_{1} d u v
\end{aligned}
$$

Because the small parameter $\left(\varepsilon^{2}\right)$ multiplies the derivatives $u^{\prime \prime}$ and $v^{\prime \prime}$, we are dealing with a singular perturbation problem. We will employ the method of matched asymptotic expansions to solve the equations (13) subject to the boundary conditions ( 7 ) and the constraint (11). 


\section{Solution of the Problem}

3a. Interior Solution

To find the solution of eq. (13) for small $\varepsilon$ we expand $u$ and $v$,

$$
\begin{aligned}
& u=u_{0}+\varepsilon^{2} u_{1}+\ldots, \\
& v=v_{0}+\varepsilon^{2} v_{1}+\ldots .
\end{aligned}
$$

Then, to lowest order in $\varepsilon$,

$$
\sigma_{1} \alpha u_{0}-\sigma_{2} v_{0}-\sigma_{1} d u_{0} v_{0}=0
$$

Thus, any nontrivial solution of the form (14) is such that

$$
u_{0}(x)=\frac{\sigma v_{0}(x)}{\alpha-d v_{0}(x)}, \quad 0<x<1 .
$$

For the higher-order coefficients we obtain a set of linear algebraic equations

$$
\begin{gathered}
\sigma_{1}\left(\alpha-d v_{0}\right) u_{i}-\left(\sigma_{2}+\sigma_{1} d u_{0}\right) v_{i}=u_{i-1}^{\prime \prime}+\sigma_{1} d \sum_{j=1}^{i-1} u_{j} v_{i-j}, \\
-\sigma_{1}\left(\alpha-d v_{0}\right) u_{i}+\left(\sigma_{2}+\sigma_{1} d u_{0}\right) v_{i}=\delta v_{i-1}^{\prime \prime}-\sigma_{1} d \sum_{j=1}^{i-1} u_{j} v_{i-j},
\end{gathered}
$$

for $i=1,2, \ldots$. This set is solvable for $\left(u_{i}, v_{i}\right)$ if the lower-order coefficients $\left(u_{0}, v_{0}\right), \ldots,\left(u_{i-1}, v_{i-1}\right)$ satisfy the solvability condition

$$
u_{i-1}^{\prime \prime}+\delta v_{i-1}^{\prime \prime}=0
$$

In particular, for $i=1$,

$$
u_{0}^{\prime \prime}+\delta v_{0}^{\prime \prime}=0
$$

From (15) we deduce an expression for $u_{0}^{\prime \prime}$ in terms of $v_{0}$ and its derivatives $v_{0}^{\prime}$ and $v_{0}^{\prime \prime}$. When we use this expression, the solvability condition (16) becomes a second order nonlinear differential equation for $v_{0}$. In fact, the 
equation is more succinctly formulated in terms of the function $w_{0}=\alpha-d v_{0}$. Name ly,

$$
-\left(c^{2}+w_{0}^{2}\right) w_{0} w_{0}^{\prime \prime}+2 c^{2}\left(w_{0}^{\prime}\right)^{2}=0
$$

where we have introduced the abbreviation

$$
c^{2}=\alpha \sigma / \delta
$$

The equation (17) can be integrated exactly. Its general solution is

$$
w_{0}(x)=a x+b \pm \sqrt{(a x+b)^{2}+c^{2}}, \quad 0<x<1,
$$

where $a$ and $b$ are arbitrary constants of integration. However, the lower sign must be rejected if $w_{0}$ is to be positive. Thus we find that, to lowest order in $\varepsilon$, the solution of (13) in the interior of the domain is given by

$$
\begin{aligned}
& u_{0}(x)=\frac{\delta}{d}\left[\sqrt{(a x+b)^{2}+c^{2}}-(a x+b)\right]-\frac{\sigma}{d}, \\
& v_{0}(x)=\frac{\alpha}{d}-\frac{1}{d}\left[a x+b+\sqrt{(a x+b)^{2}+c^{2}}\right] .
\end{aligned}
$$

We observe that, in 'general, the functions $u_{0}$ and $v_{0}$ cannot be expected to satisfy the given boundary data $(7 a),(7 b)$ for $u$ and $v$. Therefore, the expansion (14) represents an asymptotic approximation to the solution in the interior of the domain. Near each of the boundaries it is necessary to construct boundary layer expansions that must be matched with the interior solution (18). For future reference we note from (18) that

$$
\begin{aligned}
& u_{0}(x) \rightarrow u_{0}(0)=\frac{\delta}{d}\left[\sqrt{b^{2}+c^{2}}-b\right]-\frac{\sigma}{d}, \\
& v_{0}(x) \rightarrow v_{0}(0)=\frac{\alpha}{d}-\frac{1}{d}\left[b+\sqrt{b^{2}+c^{2}}\right],
\end{aligned}
$$

as $x \rightarrow 0$, and 


$$
\begin{aligned}
& u_{0}(x)+u_{0}(1)=\frac{\delta}{d}\left[\sqrt{(a+b)^{2}+c^{2}}-(a+b)\right]-\frac{\sigma}{d}, \\
& v_{0}(x)+v_{0}(1)=\frac{\alpha}{d}-\frac{1}{d}\left[a+b+\sqrt{(a+b)^{2}+c^{2}}\right],
\end{aligned}
$$

as $\mathbf{x} \rightarrow 1$

3b. Boundary Layer Solution near $x=0$

We introduce the stretched variable $z=x / \varepsilon$ and define the functions $U^{L}$ and $\mathrm{V}^{\mathrm{L}}$ by means of the relations

$$
\mathrm{U}^{\mathrm{L}}(\mathrm{z})=\mathrm{u}\left(\varepsilon_{\mathrm{z}}\right), \quad \mathrm{v}^{\mathrm{L}}(\mathrm{z})=\mathrm{v}\left(\varepsilon_{\mathrm{z}}\right)
$$

If $u$ and $v$ satisfy the equations (13), then $\mathrm{U}^{\mathrm{L}}$ and $\mathrm{v}^{\mathrm{L}}$ satisfy the equations

$$
\begin{gathered}
U^{\prime \prime}=\sigma_{1} \alpha U-\sigma_{2} V-\sigma_{1} d U V, \\
\delta V^{\prime \prime}=-\sigma_{1} \alpha U+\sigma_{2} V+\sigma_{1} d U V,
\end{gathered}
$$

for $z>0$. We will determine a solution $\left(U^{L}, V^{L}\right)$ subject to the initial conditions

$$
U(0)=\gamma_{0}, \quad V^{\prime}(0)=0
$$

cf. (7a)(7b), and the matching conditions

$$
\lim _{z \rightarrow \infty} U(z)=u_{0}(0), \quad \lim _{z \rightarrow \infty} V(z)=v_{0}(0)
$$

cf. (19a), (19b). (We drop the superscript $L$ in the remainder of this subsection.)

First, we observe that $U+\delta V$ is a linear function of $z$. Since $U$ and $V$ both tend to finite limits as $z^{+\infty}$, we must have

$$
U(z)+\delta V(z)=B, \quad z>0
$$

for some constant $B$. This relation allows us to eliminate $U$ from (21) and 
(22). The result is a nonlinear differential equation for $V$,

$$
\delta V^{\prime \prime}+\sigma_{1}\left[d \delta V^{2}-(\alpha \delta+\sigma+d \beta) V+\alpha \beta\right]=0,
$$

which must be solved subject to the initial conditions

$$
V(0)=\left(\beta-\gamma_{0}\right) / \delta, \quad V^{\prime}(0)=0
$$

The equation (25) admits two solutions that do not depend on $z$, viz. V(z) $=\mathrm{V}^{+}$and $\mathrm{V}(\mathrm{z})=\mathrm{V}^{-}$, where

$$
v^{ \pm}=\frac{1}{2 d \delta}\left[\alpha \delta+\sigma+d \beta \pm \sqrt{(\alpha \delta+\sigma+d \beta)^{2}-4 \alpha \beta \delta d}\right]
$$

Now, the initial conditions (26) imply that there is no real value of $\beta$ such that $\cdot \mathrm{V}^{+}=\left(\beta-\gamma_{0}\right) / \delta$, and that there is precisely one real value of $\beta$ such that $\mathrm{V}^{-}=\left(\beta-\gamma_{0}\right) / \delta$, namely

$$
\beta=\gamma_{0}+\frac{\alpha \delta \gamma_{0}}{\sigma+d \gamma_{0}}
$$

In that case,

$$
\begin{aligned}
& U^{L}(z)=U_{0}=\gamma_{0}, \\
& v^{L}(z)=v_{0}=\frac{\alpha \gamma_{0}}{\sigma+d \gamma_{0}} .
\end{aligned}
$$

Both matching conditions (23) are satisfied if we take

$$
\mathrm{b}=\frac{1}{2 \delta}\left[-\left(\sigma+\mathrm{d} \gamma_{0}\right)+\frac{\mathrm{c}^{2} \delta^{2}}{\sigma+\mathrm{d} \gamma_{0}}\right] .
$$

The equation (25) admits other solutions besides the ones that are independent of $z$ that we found above. Although they cannot be obtained explicitly, we can gain sufficient information about them to evaluate their relevance in the present context. If we multiply eq. (25) by V' and integrate, using the initial conditions (26), we obtain the following expression for $V^{\prime}$ in terms of $V$, 


$$
V^{\prime}= \pm\left[\frac{2}{3} d \sigma_{1}(V(0)-V) q(V)\right]^{1 / 2}
$$

where $q$ is a quadratic function of $v$,

$$
\mathrm{q}(\mathrm{V})=\mathrm{v}^{2}+\mathrm{q}_{1} \mathrm{v}+\mathrm{q}_{2}
$$

with

$$
\begin{aligned}
& \mathrm{q}_{1}=-\frac{1}{2 \mathrm{~d} \delta}\left(2 \mathrm{~d} \gamma_{0}+3 \sigma+3 \alpha \delta+d \beta\right), \\
& \mathrm{q}_{2}=-\frac{1}{2 \delta^{2} \mathrm{~d}}\left[\mathrm{~d} \beta^{2}+\left(\mathrm{d} \gamma_{0}+3 \sigma-3 \alpha \delta\right) \beta-\gamma_{0}\left(2 \mathrm{~d} \gamma_{0}+3 \sigma+3 \alpha \delta\right)\right] .
\end{aligned}
$$

The existence of non-constant solutions $V$ of the differential equation (30) is most easily studied in the phase plane $\left(V, V^{\prime}\right)$. Because of (26), we restrict our attention to trajectories that originate at $(V(0), 0)$. We observe that any trajectory emanating from the point $(V(0), 0)$ leaves the $V$-axis under a vertical tangent, cf. (30). Finally, we require that $\lim _{z \rightarrow \infty} V^{\prime}(z)=0$, so any trajectory of interest must end at some point of the $V$-axis. Now, if $q(V)>0$ for all $V$, we must have $V \leq V(0)$ for $V^{\prime}$ to be real, so $V$ is a decreasing function of $z$ near $z=0$. This rules out the upper (+) sign in (30). But then it is obvious that any trajectory, once it has left the $V$-axis at $(V(0), 0)$, stays in the lower half-plane to the left of the vertical line $V=$ $\mathrm{V}(0)$ for all $\mathrm{z}>0$ and will never return to the $\mathrm{V}$-axis. Therefore, no trajectory originating at $(\mathrm{V}(0), 0)$ meets the requirement that it end at some point of the V-axis. We conclude that (30) does not have a non-constant solution that meets all requirements. A similar conclusion holds if $q(V)<0$ for all $\mathrm{V}$.

Now, assume that $q$ has two real zeros, $v_{+}$and $V_{-}$say,

$$
v_{ \pm}=\frac{1}{4 d \delta}\left(2 d \gamma_{0}+3 \sigma+3 \alpha \delta+d \beta\right) \pm \frac{3}{4 \delta} V_{p}(\beta)
$$

where

$$
\begin{aligned}
p(\beta)= & \beta^{2}+\frac{2}{3 d}\left(2 d \gamma_{0}+5 \sigma-3 \alpha \delta\right) \beta \\
& +\frac{1}{3 d^{2}}\left(2 d \gamma_{0}+3 \sigma+3 \alpha \delta\right)\left(-2 d \gamma_{0}+\sigma+\alpha \delta\right) .
\end{aligned}
$$


Then

$$
V^{\prime}= \pm\left[\frac{2}{3} d \sigma_{1}(V(0)-V)\left(V-v_{-}\right)\left(V-v_{+}\right)\right]^{1 / 2}
$$

We investigate whether it is possible that a trajectory which originates at $(V(0), 0)$, terminates at either of the points $\left(V_{-}, 0\right)$ or $\left(V_{+}, 0\right)$.

First, we consider the case $V(0)<V_{-}<V_{+}$. Then, for $V^{\prime}$ to be real we must have $\mathrm{V}<\mathrm{V}(0)$ for $z$ near 0 , so $\mathrm{V}$ decreases, the upper $(+)$ sign for $\mathrm{V}^{\prime}$ is ruled out, and any trajectory leaving $(V(0), 0)$ stays in the lower half-plane to the left of the vertical line $V=V(0)$. No trajectory can reach either of the points $\left(V_{-}, 0\right),\left(V_{+}, 0\right)$ and, again, we conclude that there is no nonconstant solution of $(30)$ that meets all requirements.

Next, we consider the case $V_{-}<v(0)<V_{+}$. Then the condition that $V^{\prime}$ be real leads to $\mathrm{V}>\mathrm{V}(0)$ for $\mathrm{z}$ near 0 , so $\mathrm{V}$ increases and the lower (-) sign is ruled out. This time, it is indeed possible that there exists a trajectory that originates at $(V(0), 0)$ and ends at $\left(V_{+}, 0\right)$, and that lies entirely in the upper half-plane between the vertical lines $V=V(0)$ and $V=V_{+}$. Such a trajectory is traversed in the direction of increasing $v$.

Similarly, if $v_{-}<v_{+}<V(0)$ we find that there may exist a trajectory that originates at $(V(0), 0)$ and ends at $\left(V_{+}, 0\right)$, and that lies entirely in the lower half-plane between the vertical lines $V=V_{+}$and $V=V(0)$. Such a trajectory is traversed in the direction of decreasing $V$.

Hence, we can restrict ourselves to the investigation of solutions $V$ of (25) which are given implicitly by the formula (31), which satisfy the initial conditions (26), and which have the property that

$$
\lim _{z \rightarrow \infty} V(z)=V_{+}
$$

The corresponding functions $U$ are found from (24). Of course, the parameter $\beta$ is still to be determined at this point.

Next, we turn to the matching conditions (23). They become 


$$
\begin{aligned}
\beta-\delta V_{+} & =\frac{\delta}{d}\left[\sqrt{b^{2}+c^{2}}-b\right]-\frac{\sigma}{d}, \\
V_{+} & =\frac{\alpha}{d}-\frac{1}{d}\left[\sqrt{b^{2}+c^{2}}+b\right],
\end{aligned}
$$

cf. (19). Multiplying the last equation by $\delta$ and adding the two equations we see that

$$
b=\frac{1}{2}\left(\alpha-\frac{\sigma}{\delta}-\frac{d}{\delta} \beta\right)
$$

Then the second matching condition can be rewritten in the form

$$
\begin{aligned}
\sqrt{b^{2}+c^{2}} & =\alpha-b-d V+ \\
& =-\frac{1}{4}\left(\alpha-\frac{d}{\delta}\left(\beta-\gamma_{0}\right)\right)-\frac{1}{4} \frac{\sigma}{\delta}-\frac{1}{4} \frac{d \gamma_{0}}{\delta}-\frac{3}{4} \frac{d}{\delta} \sqrt{p(\beta)} .
\end{aligned}
$$

However, because $W(0)=\alpha-d V(0)=\alpha-d\left(\beta-\gamma_{0}\right) / \delta \geq 0$, the expression in the right member is certainly negative, while the expression in the left member is positive. Hence, the matching conditions cannot possibly be satisfied for any choice of $\alpha$ and $\beta$. We conclude therefore, that a solution of the differential equation (25) of the form (31) cannot be made to match the interior solution (18) and must be ruled out. Hence, the only solution in the boundary layer near $x=0$ that satisfies the initial conditions (26) and that can be matched with the interior solution (18) is the constant solution (28), with $\beta$ given by (27).

3c. Boundary Layer Solution near $x=1$

A similar analysis can be given for the boundary layer solution near $x=1$. We introduce the stretched variable $z=(1-x) / \varepsilon$ and define the functions $U^{R}$ and $V^{R}$ by the relations

$$
\mathrm{U}^{\mathrm{R}}(\mathrm{z})=\mathrm{u}\left(1-\varepsilon_{z}\right), \quad \mathrm{v}^{\mathrm{R}}(z)=\mathrm{v}\left(1-\varepsilon_{z}\right)
$$

Then $\mathrm{U}^{\mathrm{R}}$ and $\mathrm{V}^{\mathrm{R}}$ satisfy the equations (21) for $z>0$. Instead of (22) we have the initial conditions

$$
\mathrm{U}(0)=\gamma_{1}, \quad \mathrm{~V}^{\prime}(0)=0,
$$


and the matching conditions

$$
\lim _{z+\infty} U(z)=u_{0}(1), \quad \lim _{z \rightarrow \infty} V(z)=v_{0}(1)
$$

As in the previous subsection we find that the only solution $\left(U^{R}, V^{R}\right)$ is, in fact, independent of $z$ and given by

$$
\begin{aligned}
& U^{R}(z)=U_{1}=\gamma_{1}, \\
& v^{R}(z)=v_{1}=\frac{\alpha \gamma_{1}}{\sigma+d \gamma_{1}} .
\end{aligned}
$$

The matching conditions (34) are satisfied if we take

$$
a+b=\frac{1}{2 \delta}\left[-\left(\sigma+d \gamma_{1}\right)+\frac{c^{2} \delta^{2}}{\sigma+d \gamma_{1}}\right] \text {. }
$$

\section{3d. Uniform Solution}

Now that we have the interior solution to the boundary value problem (13), and the boundary layer solutions that are valid near $x=0$ and $x=1$ and that match the interior solution, we can combine the results to obtain a solution that is valid uniformly throughout the interval $[0,1]$. To lowerst order in $\varepsilon$ we have

$$
\begin{aligned}
& u(x)=\frac{\delta}{d}\left[\sqrt{(a x+b)^{2}+c^{2}}-(a x+b)\right]-\frac{\sigma}{d}, \\
& v(x)=\frac{\alpha}{d}-\frac{1}{d}\left[a x+b+\sqrt{(a x+b)^{2}+c^{2}}\right],
\end{aligned}
$$

where $a$ and $b$ are given by the expressions (29) and (36). It remains to determine $\alpha$ from the constraint (11).

Using (37b) we obtain the following expression for $\vec{v}$,

$$
\begin{aligned}
& \bar{v}=\frac{\alpha}{d}-\frac{1}{2 d}(a+2 b) \\
&-\frac{1}{2 a d}\left[\left\{(a+b) \sqrt{(a+b)^{2}+c^{2}}-b \sqrt{b^{2}+c^{2}}\right\}\right. \\
&\left.+c^{2}\left\{\ln \left(a+b+\sqrt{(a+b)^{2}+c^{2}}\right)-\ln \left(b+\sqrt{b^{2}+c^{2}}\right)\right\}\right] .
\end{aligned}
$$


We substitute $b$ and $a+b$ from (29) and (36), and use the definition $c^{2}=$ $\alpha \sigma / \delta$. We then find

$$
\bar{v}=\frac{\alpha}{d}\left[1-\frac{\sigma}{\eta_{0} \eta_{1}+\alpha \sigma \delta}\left(\rho \sigma \delta \alpha+n_{0} n_{1} \omega\right)\right],
$$

where $n_{0}, n_{1}, \rho$ and $\omega$ are given in terms of $\gamma_{0}, \gamma_{1}, \sigma$ and $d$,

$$
\begin{aligned}
\eta_{0} & =\sigma+d \gamma_{0}, \quad n_{1}=\sigma+d \gamma_{1}, \\
\rho & =\frac{1}{2}\left(n_{0}^{-1}+n_{1}^{-1}\right), \\
\omega & =\left(n_{0}-n_{1}\right)^{-1}\left(\ell n n_{0}-\ell n n_{1}\right) .
\end{aligned}
$$

When we use the expression of $\bar{v}$ in the constraint (11) we obtain a quadratic equation for $\alpha$,

$$
\mathrm{p}_{0} \alpha^{2}+\mathrm{p}_{1} \alpha+\mathrm{p}_{2}=0
$$

where the coefficients $\mathrm{p}_{i}$ are given in terms of the parameters of the problem,

$$
\begin{aligned}
& p_{0}=-\sigma \delta d \frac{\sigma^{2}+\gamma_{0} \gamma_{1} d+\frac{1}{2}\left(\gamma_{0}+\gamma_{1}\right) \sigma(1+d)}{\left(\sigma+\gamma_{0} d\right)\left(\sigma+\gamma_{1} d\right)}, \\
& p_{1}=\sigma \delta d-(1+(d-1) \sigma \omega)\left(\sigma+\gamma_{0} d\right)\left(\sigma+\gamma_{1} d\right), \\
& p_{2}=d\left(\sigma+\gamma_{0} d\right)\left(\sigma+\gamma_{1} d\right) .
\end{aligned}
$$

It remains to investigate the roots of equation (38). Because $P_{0}$ is always negative and $\mathrm{P}_{2}$ always positive, the roots are real and of opposite sign. Thus, there exists exactly one positive value of $\alpha$ such that the constraint (11) is satisfied, namely

$$
\alpha=-\frac{1}{2 p_{0}}\left[p_{1}+\sqrt{p_{1}^{2}-4 p_{0} p_{2}}\right] \text {. }
$$

This completes the solution of the problem. 


\section{Facilitation Factor}

We return to the expression (9) for the total flux of species A through the membrane. The extent to which the transport is enhanced is measured by the facilitation factor $F$,

$$
F=\delta \frac{v(0)-v(1)}{\gamma_{0}-\gamma_{1}}
$$

With the solution $v$ given by $(37 b)$, where the constants $a$ and $b$ are given in (29) and (36) and the parameter $\alpha$ is determined according to (39), we have

$$
F=\frac{\alpha \sigma \delta}{\left(\sigma+\gamma_{0} d\right)\left(\sigma+\gamma_{1} d\right)}
$$

We note the following special cases:

$$
\mathrm{d}=1\left(\mathrm{D}_{\mathrm{AB}}=\mathrm{D}_{\mathrm{B}}\right)
$$

The quadratic equation (38) reduces to

$$
(\alpha-1)\left(\sigma \delta \alpha+\left(\sigma+\gamma_{0}\right)\left(\sigma+\gamma_{1}\right)\right)=0
$$

whence $\alpha=1$, so

$$
F=F_{1}=\frac{\sigma \delta}{\left(\sigma+\gamma_{0}\right)\left(\sigma+\gamma_{1}\right)}
$$

In terms of the original parameters we have

$$
F_{1}=\frac{D_{A B}}{D_{A}} \frac{k_{1} k_{2} C}{\left(k_{2}+k_{1} C_{A}^{0}\right)\left(k_{2}+k_{1} C_{A}^{L}\right)} \text {. }
$$

This expression agrees with the ones given by Ward [5, formula (12)], Smith, Meldon and Colton [6, formula (54)], and Goddard, Schultz and Bassett [7, formula (3.30)].

$$
\mathrm{d} \approx 1\left(\mathrm{D}_{\mathrm{AB}} \approx \mathrm{D}_{\mathrm{B}}\right)
$$

A straightforward perturbation procedure yields the result 


$$
F=F_{1}\left[1-\Phi(d-1)+0\left((d-1)^{2}\right)\right]
$$

where $F_{1}$ is the value of $F$ for $d=1$, which is given by (41), and

$$
\Phi=\sigma \frac{n_{0} n_{1} \omega+\rho \sigma \delta}{n_{0} n_{1}+\sigma \delta}+\frac{\gamma_{0}}{n_{0}}+\frac{\gamma_{1}}{n_{1}}-1 .
$$

Here, $\eta_{0}, \eta_{1}, \rho$ and $\omega$ are given in terms of $\gamma_{0}, \gamma_{1}$ and $\sigma$,

$$
\begin{aligned}
\eta_{0} & =\sigma+\gamma_{0}, \quad \eta_{1}=\sigma+\gamma_{1}, \\
\rho & =\frac{1}{2}\left(\eta_{0}^{-1}+\eta_{1}^{-1}\right), \\
\omega & =\left(\eta_{0}-\eta_{1}\right)^{-1}\left(\ell_{n} \eta_{0}-\ln \eta_{1}\right) .
\end{aligned}
$$

We show that $\Phi$ is always positive.

Suppose $\gamma_{0} \geq \gamma_{1}$. Then $n_{0} \geq n_{1}$, so $\eta_{0}=n_{1}(1+z)$ for some $z \geq 0$. Thus, $\omega=\left(n_{1} z\right)^{-1} \ell_{n}(1+z)$ and $\rho=n_{1}^{-1}\left(1+\frac{1}{2} z\right)(1+z)^{-1}$. It follows from the inequality

$$
\ln (1+z) \leq z \frac{1+\frac{1}{2} z}{1+z}, \quad z \geq-1,
$$

that $\omega \leq \rho$. Hence,

$$
\Phi \geq \sigma \omega+\frac{\gamma_{0}}{n_{0}}+\frac{\gamma_{1}}{n_{1}}-1 .
$$

Next, we use the inequality

$$
\ln (1+z) \geq \frac{z}{1+z}, \quad z \geq-1
$$

to show that $\omega \geq \eta_{1}^{-1}(1+z)^{-1}=\eta_{0}^{-1}$, so

$$
\Phi \geq \frac{\sigma}{n_{0}}+\frac{\gamma_{0}}{n_{0}}+\frac{\gamma_{1}}{n_{1}}-1=\frac{\gamma_{1}}{n_{1}}>0 .
$$

Similarly, one shows that $\Phi>0$ if $\gamma_{0} \leq \gamma_{1}$, so $\Phi$ is indeed always positive.

The coefficient $\Phi$ may be called the enhancement coefficient. It measures the effect of different diffusion coefficients for the nonvolatile species $B$ 
and $A B$ on the facilitation factor $F$, relative to the situation where $B$ and $A B$ have equal diffusion coefficients. As can be seen from (42), the facilitation factor $F$ is enhanced when $d<1$ (i.e. $D_{B}>D_{A B}$ ), inhibited when $d>1$ (i.e. $\mathrm{D}_{\mathrm{AB}}>\mathrm{D}_{\mathrm{B}}$ ). 


\section{Notation}

$A, B, A B \quad$ molecular species

$D_{A}, D_{B}, D_{A B}$ diffusion coefficients

$C_{A}, C_{B}, C_{A B}$ molar concentrations

$x^{*}$

coordinate perpendicular to the membrane surfaces

L

thickness of the membrane

$\mathrm{k}_{1}, \mathrm{k}_{2}$

$\mathrm{C}_{\mathrm{A}}^{0}, \mathrm{C}_{\mathrm{A}}^{\mathrm{L}}$

$\mathrm{C}$

forward $(\rightarrow)$ and backward $(*)$ reaction rates in (1)

specified molar concentrations of $A$ at $x^{*}=0$ and $x^{*}=L$

specified total molar concentration of $B$ and $A B$ inside the membrane

$\mathrm{N}_{\mathrm{A}}$

total flux of species $A$

$\mathrm{x}$

dimensionless coordinate

$\mathrm{u}, \mathrm{v}, \mathrm{w}$

dimensionless molar concentrations

$\overline{\mathrm{v}}$

integral of $\mathrm{v}$ over $[0,1]$

$\gamma_{0}, \gamma_{1}$

boundary values of $u$

$\kappa_{1}, \kappa_{2}$

dimensionless reaction rates

$\delta, \mathrm{d}$

ratios of diffusion coefficients

$\alpha, \beta, a, b$

constants

$\varepsilon$

small parameter, $0<\varepsilon<1$

$\sigma_{1}, \sigma_{2}$

dimensionless reaction rates; $\sigma_{i}=\kappa_{i} / \varepsilon^{2}$

$\sigma$

ratio of reaction rates; $\sigma=\sigma_{2} / \sigma_{1}=\kappa_{2} / \kappa_{1}$

c parameter; $c^{2}=\alpha \sigma / \delta$

$\mathrm{u}_{0}, \mathrm{u}_{1}, \ldots \quad$ coefficients of $u$ in the interior solution

$\mathrm{v}_{0}, \mathrm{v}_{1}, \ldots \quad$ coefficients of $\mathrm{v}$ in the interior solution

$\mathrm{U}^{\mathrm{L}}, \mathrm{V}^{\mathrm{L}}$

boundary layer solution near $x=0$

$\mathrm{U}^{\mathrm{R}}, \mathrm{V}^{\mathrm{R}}$

boundary layer solution near $x=1$

$n_{0}, n_{1}$

$\rho, \omega$

parameters

$\mathrm{P}_{0}, \mathrm{P}_{1}, \mathrm{P}_{2} \quad$ parameters

$\mathbf{q}_{1}, \mathbf{q}_{2} \quad$ parameters

F

facilitation factor

$\mathrm{F}_{1}$

value of facilitation factor when $D_{A B}=D_{B}$

$\Phi$

enhancement (inhibition) coefficient in the facilitation 


\section{References}

[1] Matulevicius, E. S., and N. N. Li, "Facilitated Transport through Liquid Membrances," Separation and Purification Methods, 4 (1), 73-96 (1975).

[2] Murray, J. D., Lectures on Nonlinear Differential Equation Models in Biology, Oxford Univ. Press (1977).

[3] Scholander, P. F., "Oxygen Transport through Hemoglobin Solutions," Science, 131, 585 (1960).

[4] Wittenberg, J. B., "Oxygen Transport -- A New Function Proposed for Myoglobin," Abstract, Biol. Bu11., 1171, 402-403 (1959).

[5] Ward, W. J., "Analytical and Experimental Studies of Facilitated Transport," AIChE Journal, 16, 405-410 (1970).

[6] Smith, K. A., Meldon, J. H., and Colton, C. K., "An Analysis of CarrierFacilitated Transport," AIChE Journal, 19, 102-111 (1973).

[7] Goddard, J. D., Schultz, J. S., and Bassett, R. J., "On Membrane Diffusion with Near-Equilibrium Reaction," Chem. Engin. Science, 25, 665-683 (1970).

[8] Goddard, J. D., Schultz, J. S., and Suchdeo, S. R., "Facilitated Transport via Carrier-Mediated Diffusion in Membranes, II," AIChE Journal 20, 625-645 (1974).

[9] Aris, R., The Mathematical Theory of Diffusion and Reaction in Permeable Catalysts, Oxford Univ. Press (1975). 
Distribution for ANL-80-71

Internal:

J. M. Cook

G. T. Garvey

H. G. Kaper (25)

G. K. Leaf (5)

B. J. Matkowsky (5)

P. C. Messina

R. J. Royston
L. Phebus (7)

A. B. Krisciunas

ANL Contract File

ANL Libraries

TIS Files (6)

\section{External:}

DOE-TIC, For distribution per UC-32 (183)

Manager, Chicago Operations and Regional Office, DOE-CORO

Chief, Office of Patent Counsel, DOE-CORO

President, Argonne Universities Association

Applied Mathematics Division Review Committee:

G. Estrin, U. California, Los Angeles

W. M. Gentleman, U. Water 100

J. M. Ortega, U. Virginia

E. N. Pinson, Bell Telephone Labs

S. Rosen, Purdue U.

M. F. Wheeler, Rice U.

D. M. Young, Jr., U. Texas at Austin

J. Corones, Iowa State U.

J. H. Ferziger, Stanford U.

J. Glimm, Rockefeller U.

J. Grasman, MC Amsterdam

E. W. Larsen, LASL

P. Nelson, Texas Tech

C. V. Pao, North Carolina State U.

A. D. Solomon, ORNL

H. D. Thames, U. of Texas, Houston 